\title{
IVIG in Adult onset Stills Disease
}

\author{
Gregory Williamson, Elizabeth Profeta and Alexander Mayers* \\ Penn State College of Medicine, USA
}

\section{Case presentation}

Mr. H is a 57-year-old male with Adult Onset Stills Disease, CKD, IVIG dependent smoldering myeloma, HTN, and iatrogenic hypoparathyroidism admitted with persistent fevers, chills, malaise, confusion, leukocytosis, and acute on chronic renal failure. On exam he demonstrated an evanescent, salmon-colored, macular rash over the proximal arms, shoulders, and axilla. His infectious work-up revealed negative blood cultures, unremarkable $\mathrm{CXR}$, and negative urine legionella antigen. CBC revealed leukocytosis, and vitals displayed hypotension and tachycardia. Although he met SIRS criteria indicative of sepsis, we determined he had a Still's flare masquerading as sepsis. Rheumatology recommended prednisone $60 \mathrm{mg}$ qd, up from his usual $7 \mathrm{mg}$, and to withhold IVIG. Oncology recommended a myeloma work-up, which revealed SPEP: IgM <25, IgG 2120, IgA 50, and kappalambda ratio 10.9. Clinically, he had several encephalopathic and febrile episodes, but head CT showed no acute intracranial abnormality. With IV steroids, his leukocytosis decreased from 34 to 15 , he defervesced, and his encephalopathy resolved. He was discharged with prednisone $50 \mathrm{mg}$ qd on hospital day 10.

\section{Discussion}

Still's disease is a rare condition, with uncertainty regarding a definitive treatment. Traditional treatment for Stills has centered around NSAIDs, corticosteroids, and DMARDs [1-6]. However, IVIG is frequently used in the treatment of both Still's and myeloma $[2,3]$. Additionally, it has minimal side effects and can reduce steroid requirements, thus sparing individuals from the side effects of high dose steroids [7]. Mr. H was receiving IVIG every 5 weeks for his myeloma with the next dose due on the date of admission. However, it was withheld in light of his clinically deteriorating picture and was given high dose steroids instead. This inspired the question: "In a patient with a Still's flare and concomitant smoldering myeloma, would IVIG remain an effective treatment for Still's flare, as compared to high dose prednisone?".

\section{Summary}

An unblinded research trial showed IVIG could represent a potential alternative to steroid therapy in patients with Stills disease refractory to NSAIDs [4].

1. A small study found no difference in clinical features or prognosis between patients with refractory Still's flare that received IVIG and those who did not. However, in the subset of patients who were responsive to IVIG, the frequency of good prognosis was significantly higher [5].

2. The most recent international guidelines for management of sepsis recommend against the use of IVIG in patients with sepsis [8].

3. Relative contraindications to IVIG include IgA deficiency and renal impairment. Additionally, IVIG can cause acute renal dysfunction [9].

\section{Conclusion}

The only relative contraindication to the use of IVIG in Mr. H was acute on chronic kidney failure. If he truly had sepsis, IVIG would not have been an appropriate part of his treatment. However, once sepsis was ruled out and kidney function returned to baseline, IVIG may have been considered as a treatment for his Still's and myeloma instead of, or in addition to, prednisone.

\section{Contributors}

Kevin Parks and Karthik Sabapathy.

\section{References}

1. Efthimiou P, Paik K, Bielory L (2006) Diagnosis and management of adult onset Still's disease. Ann Rheum Dis 65: 564-572.

2. Jolles S, Sewell W, Misbah S (2005) Clinical uses of intravenous immunoglobulin British Society for Immunology, Clinical and Experimental Immunology 142: 1-11.

3. Mahmud T, Hughes R (1999) Intravenous immunoglobulin in the treatment of refractory adult Still's disease. J Rheumatol 26: 2067-2068.

4. Vignes S (1998) Intravenous immunoglobulin in adult Still's disease refractory to nonsteroidal anti-inflammatory drugs. Clin Exp Rheumatol 16: 295-298.

5. Kim A, Sung M, Suh H (2012) Therapeutic responses and prognosis in adult-onset Still's disease. Rheumatol Int 32: 1291.

6. Agha-Abbaslou M (2017) Adult-onset still's disease: still a serious health problem (a case report and literature review). Am J Case Rep 18: 119-124.

7. Agranov K, Khattri S, Zandman-Goddard G (2015) The role of intravenous immunoglobins in the treatment of rheumatoid arthritis. Autoimmunity Reviews 651658

8. Rhodes A (2017) Surviving sepsis campaign: international guidelines for manegement of sepsis and septic shock. Society of Critical Care Medicine 45: 508.

9. Immune globulin: drug information.
Copyright: (C)2018 Williamson G. This is an open-access article distributed under the terms of the Creative Commons Attribution License, which permits unrestricted use, distribution, and reproduction in any medium, provided the original author and source are credited.
${ }^{*}$ Correspondence to: Alexander Mayers, Penn State College of Medicine, M3, F, 2d Lt, USA, Tel: 717515 3100; E-mail: alexjmayers@gmail.com

Received: April 25, 2018; Accepted: May 11, 2018; Published: May 14, 2018 